\title{
Comportamientos de riesgo de suicidio y calidad de vida relacionada con la salud en estudiantes que ingresaron a una universidad mexicana
}

\author{
Suicide-related behavior and health-related quality of life among \\ first-year university students in a Mexican university
}

Carlos Alejandro Hidalgo-Rasmussen (https://orcid.org/0000-0002-5287-2076) ${ }^{1}$

Yolanda Viridiana Chávez-Flores (https://orcid.org/0000-0003-0613-167X) ${ }^{2}$

Libia Yanelli Yanez-Peñúñuri (https://orcid.org/0000-0003-4682-5123) ${ }^{3}$

Sergio R Muñoz Navarro (https://orcid.org/0000-0001-8383-6599) ${ }^{4}$

${ }^{1}$ Centro de Investigación en Riesgos y Calidad de Vida, Departamento de Promoción, Preservación y Desarrollo de la Salud, Universidad de Guadalajara. Centro de Estudios

Avanzados, Universidad de Playa Ancha. Av. Juárez 976, col Americana. $44160 \mathrm{Cd}$. Guzmán Jalisco México. carlos.hidalgo@academicos. udg.mx

${ }^{2}$ Escuela de Ciencias de la Salud Valle de las Palmas, Universidad Autónoma de Baja California. Tijuana México.

${ }^{3}$ Departamento de Ciencias Sociales, Universidad de Sonora. Sonora México. ${ }^{4}$ Departamento de Salud Pública, Facultad de Medicina, Universidad de La Frontera. Temuco Chile.

\begin{abstract}
The objective was to determine if suicide-related behavior (SRB) and health-related quality of life (HRQL) are associated and whether this association is independent of substance use, violence, and sociodemographic variables. It involved a cross-sectional study with 1,229 Mexican university students: $62.4 \%$ women; $37.6 \%$ men; age 18.2 .6 years. The YRBS and KISDSCREEN questionnaires were applied. The results were as follows: $14.2 \%$ students reported despair; $4.7 \%$ had suicidal ideation (SI); $4 \%$ had suicidal tendencies (ST); and 2.3\% had attempted suicide. Multiple logistic regression models show that despair increased the possibility of lower scores in all HRQL domains except economic resources with odds ratio OR (CI 95\%) ranging from 1.5 (1.0-2.3) for autonomy to 4.6 (3.1-6.8) for state of mind. The SI increased the possibility of a lower score in relationships with parents and family life (3.9, 1.7-8.9) and in friends and social support (2.9, 1.3-6.4). The ST increased the possibility for lower physical $(2.7,1.2-6.1)$ and psychological well-being (3.1, 1.3-7.2). HRQL is associated with SRB among Mexican students even after adjustment for substance use and violence. It highlights the fact that despair is negatively associated with different domains of the HRQL of the students.
\end{abstract}

Key words Attempted suicide, Suicidal ideation, Quality of life, Adolescent, Students
Resumen El objetivo fue determinar si existe asociación entre comportamientos de riesgo de suicidio (CRS) y menor calidad de vida relacionada con la salud (CVRS) y si esta asociación es independiente del consumo de sustancias, violencia y variables sociodemográficas. Estudio transversal, con 1229 estudiantes universitarios mexicanos; $62.4 \%$ mujeres $y 37.6 \%$ hombres, edad 18.2 \pm .6 años. Se aplicaron los instrumentos YRBS $y$ KIDSCREEN-52. Presentaron desesperanza $14.2 \%$, ideación suicida (IS) $4.7 \%$, planeación suicida (PS) $4.0 \%$ e intento suicida $2.3 \%$. Modelos de regresión logística múltiple muestran que la desesperanza aumentó la posibilidad de menor puntuación en todos los dominios de CVRS excepto en recursos económicos, con odds ratio $O R$ (IC95\%) que van de 1.5 (1.0-2.3) para autonomía, hasta 4.6 (3.1-6.8) para estado de ánimo. La IS incrementó la posibilidad de menor puntuación en relación con los padres y vida familiar (3.9, 1.7-8.9) y en amigos y apoyo social (2.9, 1.3-6.4). La PS aumentó la posibilidad de menor bienestar fisico (2.7, 1.2-6.1) y psicológico (3.1, 1.3-7.2). En estudiantes mexicanos la CVRS se asocia a los CRS aún después de ajustar por consumo de sustancias y violencia. Se destaca que la desesperanza se asocia negativamente a los distintos dominios de la CVRS de los estudiantes.

Palabras clave Intento de suicidio, Ideación suicida, Calidad de vida, Adolescente, Estudiantes 


\section{Introducción}

El suicidio es un grave problema de salud pública que genera un impacto psicológico, social $\mathrm{y}$ financiero en las familias y comunidades de todo el mundo․ En México, la tasa de suicidio en el año 2014 fue de 5.2 muertes por cada 100 mil habitantes, siendo 4 de cada 10 suicidios en personas de 15 a 29 años. En instituciones de salud pública, el $23 \%$ de los egresos hospitalarios atendidos por causa de lesiones autoinflingidas, sin considerar los egresos por defunción, fueron en el grupo de 15 a 19 años².

Cabe mencionar que el comportamiento suicida es resultado de una compleja interacción entre factores biológicos, genéticos, psicológicos, sociales, culturales y medioambientales ${ }^{3}$. En el modelo de desarrollo transaccional para la conducta suicida del joven, se describen varios de esos factores de riesgo $\mathrm{O}^{4}$, entre los cuales se incluyen la desesperanza como antecedente de la depresión ${ }^{5}$, la ideación suicida, la planeación suicida, y el intento suicida ${ }^{6}$. Estos factores son comportamientos de riesgo a la salud por estar en cadena causal con el suicidio ${ }^{7}$ y se han asociado a uso de sustancias, violencia y ser mujer ${ }^{8-11}$.

Por su parte la calidad de vida relacionada con la salud (CVRS), considerada como la salud física, mental y social evaluada individual o grupalmente ${ }^{12}$, se puede modificar cuando hay acontecimientos o comportamientos que impactan la vida de las personas ${ }^{13-16}$, tales como los comportamientos de riesgo de suicidio (CRS). Una menor calidad de vida se ha asociado también al consumo de tabaco ${ }^{17}$, alcohol ${ }^{18,19}$, y marihuana ${ }^{20}$; a haber sido abusado sexualmente ${ }^{21}$, a la violencia física ${ }^{22}$ and the extent to which such differences might be associated with social circumstances and lifestyle conditions. Finally, we explored the HRQoL consequences of exposure to violence in a longer time perspective. Methods: We used data from self-completed questionnaires in two Danish nationally representative, cross-sectional health interview surveys. Exposure to violence was indicated through specific survey questions (Straus' conflict tactics scale, no trabajar ${ }^{23}$, ser mujer y tener mayor edad ${ }^{24}$, discapacidad ${ }^{25} \mathrm{y}$ a menor nivel socioeconómico ${ }^{26}$.

Si bien existen estudios de CRS en estudiantes, se han realizado pocos en universitarios mexicanos ${ }^{27-31}$ y su asociación con calidad de vida solo se ha estudiado en estudiantes de preparatoria $^{32}$, lo que demuestra la necesidad de conocer cómo se asocian en esta población en específico.

Ante este contexto, el objetivo de este estudio fue determinar si existe asociación entre CRS y menor CVRS en estudiantes universitarios mexicanos, así como determinar si esta asociación es independiente del consumo de tabaco, alcohol y marihuana; del abuso sexual y la violencia física en el noviazgo, del trabajo, género, edad, discapacidad y nivel socioeconómico.

\section{Material y método}

Se realizó un estudio con diseño transversal y analítico en el año 2016, en estudiantes que se encontraban en el curso de inducción a su primer semestre de Universidad. La cantidad de estudiantes matriculados fue de 1887 y de ellos 1670 estudiantes que se presentaron al curso de inducción después de aceptar el consentimiento informado contestaron los instrumentos en aulas de cómputo. De estos, 1283 cumplieron el criterio de inclusión de tener una edad de 17 a 19 años. Después de considerar la calidad de los datos, 1229 participantes fueron incluidos en el análisis.

Para evaluar el CRS, el consumo de sustancias y la violencia, se utilizó una versión adaptada del instrumento Youth Risk Behavior Survey $(Y R B S)^{33}$, que fue utilizada en un estudio previo $^{32}$. Del instrumento se utilizaron cuatro ítems referidos a CRS presentados durante los últimos 12 meses. Las preguntas fueron 1.- ¿Te sentiste muy triste o sin esperanza casi todos los días por dos semanas consecutivas o más, al punto que dejaste de hacer algunas actividades usuales? 2.$¿$ Alguna vez consideraste seriamente intentar suicidarte? 3.- ¿Hiciste un plan sobre cómo intentarías suicidarte? y 4.- ¿Cuántas veces intentaste realmente suicidarte? Las opciones de respuesta para las preguntas sobre desesperanza, ideación y planeación suicida fueron sí/no y para la pregunta sobre intento suicida fueron: ninguna vez, 1 vez, 2 ó 3 veces, 4 ó 5 veces y 6 ó más veces.

Se preguntó para los últimos 30 días la cantidad de cigarrillos de tabaco consumidos por día, las veces de consumo abusivo de alcohol (5 o más vasos o latas de bebidas alcohólicas seguidas, es decir, en un par de horas). Para consumo de marihuana se preguntó las veces que la consumió en la vida. Para abuso sexual y violencia física se preguntó en los últimos 12 meses las veces que se le forzó a tener conductas sexuales, así como las veces que fue lastimada físicamente por parte de una persona con quien tenía una relación sentimental o con quien estaba saliendo.

Del instrumento se ha reportado su confiabilidad mediante kappa, obteniendo para la pregunta sobre desesperanza porcentajes de acuerdo moderados $(56.4 \%)$, y porcentajes sustanciales 
para ideación suicida $(74.3 \%)$, planeación suicida $(66.6 \%)$ intento suicida $(72.7 \%)$, consumo de tabaco $(83.5 \%)$, alcohol $(67.6 \%)$, marihuana $(89.8 \%)$, haber sido forzado a relación sexual (65.8\%) y haber sido víctima de violencia física en el noviazgo $(53.6 \%)^{34}$. La validez del cuestionario ha sido considerada ${ }^{35}$. En el presente estudio la consistencia interna de los cuatro ítems de comportamiento suicida evaluada mediante $\mathrm{Ku}$ der-Richardson (KD-20) fue de .67.

Para evaluar la CVRS se utilizó el cuestionario KIDSCREEN-52 en su versión adaptada para México $^{36}$. Este instrumento indaga sobre la calidad de vida experimentada en los últimos 7 días y está compuesto de 52 ítems que forman diez dominios con opciones de respuesta en una escala Likert de cinco puntos que va de menor a mayor frecuencia o intensidad. Existe evidencia del adecuado funcionamiento de los ítems en cuanto a su validez y confiabilidad ${ }^{35,37}$. Para este estudio el alfa de Cronbach por dimensión fue: bienestar físico .81; bienestar psicológico .88; estado de ánimo .87; autopercepción .71; autonomía .88; relación con los padres y vida familiar .90; amigos y apoyo social .85; entorno escolar .88; aceptación social .70 y recursos económicos .85 .

Para las variables sociodemográficas, se consideró género, trabajo (sí/no), edad, discapacidad (sí/no) y nivel socioeconómico, que se obtuvo considerando la ocupación y escolaridad de la persona que más aportaba económicamente en su hogar ${ }^{38}$.

El presente estudio es distinto a uno previo realizado en México $^{32}$ en cuanto a población de estudio: estudiantes universitarios con media de edad 18.2 \pm .57 versus estudiantes de preparatoria con media de edad $15.4 \pm .87$; también es distinto en cuanto al instrumento utilizado: KIDSCREEN-52 que es un instrumento de CVRS de diez dominios versus Youth Quality of Life-Research Instrument (YQOL-R) que es un instrumento de calidad de vida general de cuatro dominios; finalmente en el presente estudio no sólo se controló por la variable género como en el anterior estudio, sino también por variables como el abuso sexual, la violencia física y el uso de drogas, variables que se sabe están asociadas a la calidad de vida.

\section{Análisis de datos}

Las opciones de respuesta para la pregunta sobre intento suicida fueron recodificadas de la siguiente manera: $0=$ ninguna vez y $1=1$ o más in- tentos. Las variables de control se recodificaron como: consumo de tabaco $>6$ cigarrillos diarios $=1, \leq 5=0$; consumo de alcohol $>5$ bebidas en 2 horas $=1, \leq 4=0$; consumo de marihuana $\geq 3$ veces $=1, \leq 2=0$; trabajo sí $=1$, no $=0$; género mujer $=1$, hombre $=0$; edad $15-17=1,18$ y 19 $=0$; discapacidad sí $=1$, no $=0$ y nivel socioeconómico medio bajo y trabajador $=1$, medio alto $\mathrm{y}$ alto $=0$; abuso sexual sí $=1$, no $=0$; violencia física en el noviazgo sí $=1$, no $=0$.

Para determinar los valores inferiores y superiores de los dominios de CVRS se consideró un valor de CVRS inferior cuando obtuvo una puntuación igual o menor a 42 dado que en estudios clínicos ${ }^{39}$ including methods of determining responsiveness and minimal important differences. For clinical trials, instruments need to be based on a clear conceptual framework, have evidence supporting content validity and acceptable psychometric qualities. The measures must also have evidence documenting responsiveness and interpretation guidelines (i.e., minimal important difference se ha reportado como punto de corte relacionado con un tamaño de efecto importante.

Se utilizó un modelo de regresión logística múltiple para el estudio de la asociación entre cada CRS según cada una de los dominios de calidad de vida. Para ello se hizo una selección previa de variables de control que entrarían en el modelo mediante análisis de regresión logística estratificados. Después se realizaron modelos para cada dimensión de CVRS, las variables de CRS y las variables de control seleccionadas (consumo de sustancias, violencia y variables sociodemográficas). Se obtuvo el valor de $\mathrm{P}$ y el Odds Ratio (OR) e intervalos de confianza IC95\%. En todos los análisis se consideró un valor de significación de 5\%. Se utilizó el software estadístico Stata/SE 14.0 (StataCorp., College Station, TX, USA).

\section{Ética}

El Proyecto de investigación fue autorizado por el Comité de Bioética de la Universidad de Guadalajara. Se cumplieron los principios éticos contenidos en la Declaración de Helsinki de las Pautas éticas Internacionales para la Investigación Biomédica en Seres Humanos (1964, reformulada en 2013), de la Asociación Médica Mundial ${ }^{40}$. Esta investigación se consideró de "riesgo mínimo” según la Ley General de Salud de México. Los participantes fueron informados y otorgaron su consentimiento, la aplicación fue voluntaria y los datos se manejaron confidencialmente. 


\section{Resultados}

La muestra estuvo compuesta por 1229 estudiantes mexicanos de nuevo ingreso a una universidad mexicana en el año 2016, de los cuales 767 (62.4\%) eran mujeres y 462 (37.6\%) hombres. La media de edad fue de 18.2 años (DE= .6). La mayoría de los estudiantes eran solteros, de 18 a 19 años de edad, no trabajaban, de nivel socioeconómico medio alto y alto y sin discapacidad (Tabla 1).

En cuanto a las variables de control, consumieron 2 o más cigarros en los últimos 30 días 4.1\% de los participantes; consumieron cocaína alguna vez, $1.5 \%$; consumieron cinco o más bebidas en los últimos 30 días, 31.2\%; consumieron marihuana 3 o más veces en la vida $4.6 \%$; fueron víctimas de abuso sexual alguna vez, 1.1\%; y de violencia física de noviazgo en los últimos 12 meses $5.8 \%$.

La desesperanza fue el CRS más prevalente, mientras que la ideación suicida, la planeación suicida y el intento suicida se presentaron en menos del 5\% de los participantes. Los dominios de CVRS con la mayor proporción de participantes que tuvieron puntuación inferior fueron el de recursos económicos, el de estado de ánimo y el de

Tabla 1. Datos sociodemográficos de estudiantes mexicanos que ingresaron a un campus universitario en el año $2016(n=1229)$.

\begin{tabular}{|c|c|c|c|}
\hline & Variables & $\mathbf{n}$ & $\%$ \\
\hline \multicolumn{4}{|l|}{ Sexo } \\
\hline Mujeres & & 767 & 62.4 \\
\hline Hombres & & 462 & 37.6 \\
\hline \multicolumn{4}{|l|}{ Edad } \\
\hline & 17 años & 95 & 7.7 \\
\hline & 18 a 19 años & 1134 & 93.3 \\
\hline \multicolumn{4}{|c|}{ Estado civil } \\
\hline & Soltero(a) & 1217 & 99.0 \\
\hline & $\begin{array}{l}\text { Casado(a) o unión } \\
\text { Libre }\end{array}$ & 12 & 1.0 \\
\hline \multicolumn{4}{|l|}{ Trabajo } \\
\hline & Sí & 374 & 30.4 \\
\hline & No & 855 & 69.6 \\
\hline \multicolumn{4}{|c|}{ Nivel socioeconómico } \\
\hline & Medio alto y alto & 688 & 56.0 \\
\hline & Medio bajo y trabajador & 541 & 44.0 \\
\hline \multicolumn{4}{|c|}{ Discapacidad } \\
\hline & Sí & 72 & 5.9 \\
\hline & No & 1157 & 94.1 \\
\hline
\end{tabular}

bienestar físico, mientras que el dominio entorno escolar obtuvo la menor proporción (Tabla 2).

En las Tablas 3, 4 y 5 se puede observar que la desesperanza aumentó la posibilidad de una puntuación menor en todos los modelos de CVRS excepto en recursos económicos con OR que van de 1.5 (IC95\%1.0-2.3) para autonomía hasta 4.6 (IC95\%3.1-6.8) para estado de ánimo. La ideación suicida aumentó la posibilidad de una puntuación menor en el dominio de relación con padres y vida familiar con OR de 3.9 (IC95\% 1.7-8.9) y en el dominio de amigos y apoyo social de 2.9 (IC95\%1.3-6.4). La planeación suicida aumentó la posibilidad de un bienestar físico menor con un OR de 2.7 (IC95\%1.2-6.1) y de un bienestar psicológico menor con un OR de 3.1 (IC95\%1.3-7.2).

\section{Discusión}

El propósito de este estudio fue determinar si existía asociación entre CRS y menor CVRS en estudiantes universitarios mexicanos, así como determinar si esta asociación era independiente del consumo de tabaco, alcohol y marihuana; del abuso sexual y la violencia física en el noviazgo,

Tabla 2. Estudiantes mexicanos que ingresaron a un campus universitario en el año 2016, que obtuvieron una puntuación inferior en cada uno de los dominios de CVRS y que presentaron comportamientos de riesgo de suicidio $(\mathrm{n}=1229)$.

\begin{tabular}{lll}
\hline \multicolumn{1}{c}{ Variables } & n & $\%$ \\
\hline $\begin{array}{l}\text { Comportamientos de riesgo de } \\
\text { suicidio }\end{array}$ & & \\
Desesperanza & 174 & 14.2 \\
Ideación suicida & 58 & 4.7 \\
Planeación suicida & 49 & 4.0 \\
Intento suicida & 28 & 2.3 \\
Dominios de CVRS & & \\
Bienestar físico & 497 & 40.4 \\
Bienestar psicológico & 223 & 18.1 \\
Estado de ánimo & 508 & 41.3 \\
Autopercepción & 293 & 23.8 \\
Autonomía & 238 & 19.4 \\
Relación con los padres y vida familiar & 191 & 15.5 \\
Amigos y apoyo social & 291 & 23.7 \\
Entorno escolar & 44 & 3.6 \\
Aceptación social (Bullying) & 287 & 23.4 \\
Recursos económicos & 656 & 53.4 \\
\hline Nota: Para los dominios de calidad de vida relacionada con la \\
salud (CVRS) se consideró inferior una puntuación igual o \\
menor a 42.
\end{tabular}


del trabajo, género, edad, discapacidad y nivel socioeconómico. Se encontraron asociados nueve dominios de CVRS a la desesperanza; la ideación a dos dominios; la planeación a dos y estas asociaciones fueron independientes de las variables de control. No obstante, el intento suicida no se asoció a ningún dominio de CVRS.

La desesperanza fue el CRS que se asoció con todos los dominios de la CVRS excepto recursos económicos, lo cual cobra sentido considerando a los estudios que reportan que la desesperanza puede ser uno de los predictores más importantes para la consumación del suicidio ${ }^{41}$.

La desesperanza se ha visto asociada al bienestar físico en diversos estudios en adolescentes; por ejemplo, un estudio encontró que quienes realizaron alguna actividad física aeróbica o de resistencia al menos una vez por semana, presentaron menor riesgo de desesperanza ${ }^{42}$.

La asociación entre desesperanza y el estado de ánimo es consistente con una investigación previa que reportó que experimentar excesivas emociones negativas y escasez de emociones positivas, pueden llevar a experimentar desesperan$\mathrm{za}^{43}$.

Por otra parte, la relación entre desesperanza y autopercepción es consistente con lo reportado en un estudio sobre desesperanza y sentirse no atractivo ${ }^{44}$, así como con tener una visión negativa de sí $\mathrm{mismo}^{45}$.

También son congruentes los resultados de la presente investigación, con los de estudios que encontraron asociada la desesperanza al bienestar psicológico en población de adolescentes ${ }^{46} \mathrm{y}$ a una relación insatisfactoria con figuras relevantes en la adolescencia, como son los padres ${ }^{45,47,48}$.

Nuestros resultados, van en el mismo sentido que los de una investigación con jóvenes de 14 a 22 años en la que se reporta una correlación pequeña de la desesperanza con falta de apoyo de los profesores y con falta de respeto a la perspectiva de los adolescentes por parte de sus profesores, así como una correlación media con clima escolar no respetuoso ${ }^{49}$.

Sin embargo, a diferencia de lo encontrado en nuestro estudio en el cual se asoció la desesperanza a una puntuación menor en la dimensión de amigos y apoyo social, en otros estudios no se encontró relacionada la desesperanza con la pérdida de amigos ${ }^{45}$.

El efecto que tiene la desesperanza sobre tantas dimensiones de la vida de la persona requiere especial atención y coincidimos con lo que ya se ha señalado en cuanto a que la desesperanza es un punto central para la prevención del suicidio.

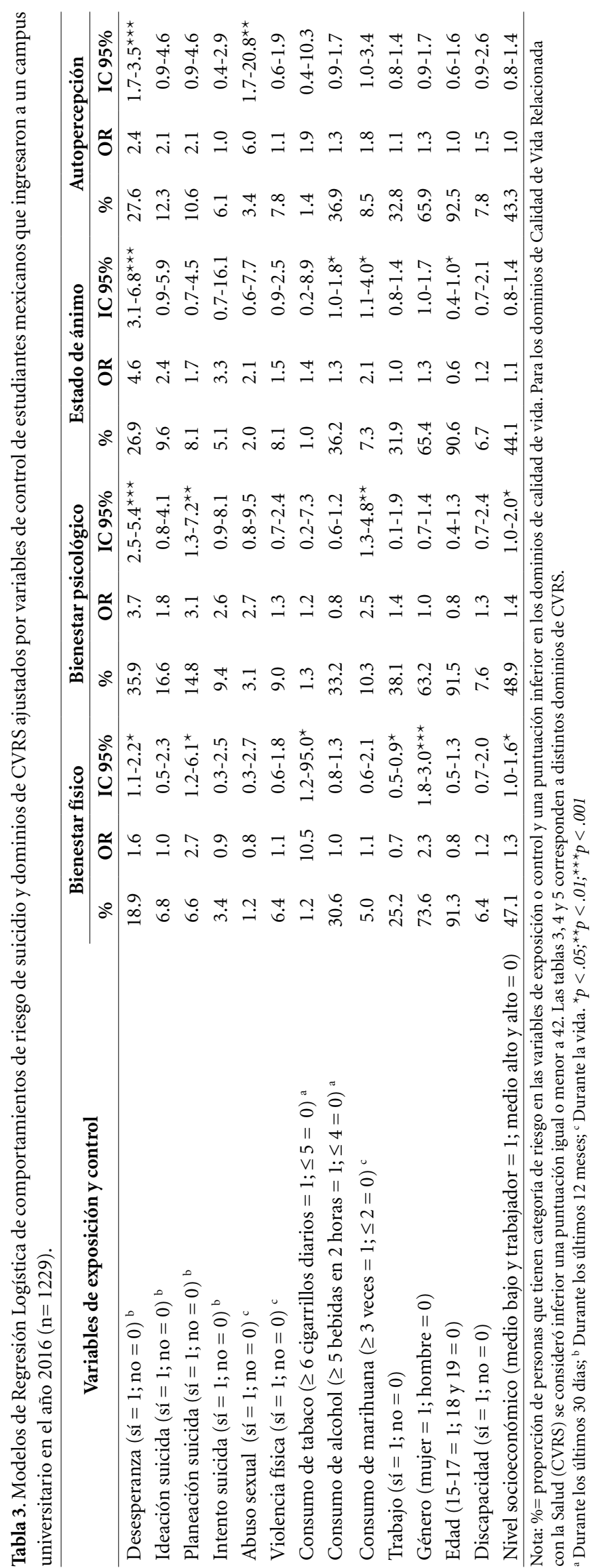


Los otros dos CRS que resultaron asociados con alguno de los dominios de CVRS fueron la ideación y la planeación suicida. La ideación se asoció con los dominios de relación con los padres y vida familiar, la de amigos y apoyo social y con estado de ánimo. Probablemente en una importante cantidad de casos la ideación suicida siga a una relación deteriorada con los padres y a la falta de apoyo social ${ }^{50}$ que experimenta el adolescente en sucesos adversos de su vida que cobran mucha importancia en esta etapa, tal como la ruptura de relaciones amorosas o de amistad. De hecho en un estudio se reportó que el aislamiento social y el tener amigos que no son amigos entre sí, está asociado a mayor probabilidad de ideación suicida en las mujeres ${ }^{51}$.

La distinción entre ideación suicida y planeación suicida no siempre es fácil ${ }^{52}$ a menos que la persona desarrolle planes muy concretos al respecto. La planeación suicida solo se asoció a los dominios de bienestar físico y bienestar psicológico de CVRS, mientras que el intentar suicidarse no se asoció significativamente con puntuaciones menores de CVRS en ninguno de los dominios.

Es de llamar la atención que la desesperanza siendo un factor distal respecto al suicidio, esté asociado a casi todos los dominios de CVRS mientras que los demás comportamientos que se podrían asumir más proximales ${ }^{4}$, se encuentren asociados solo a dos dominios o a ninguno. Una posible explicación podría ser que a medida que la persona avanza desde la desesperanza al intento suicida, pudiera estar menos interesada en distintos dominios de su vida lo que llevaría a que cuando contestara el cuestionario pusiera menos interés en dar una contestación precisa en ese momento. A diferencia del presente estudio, uno antecedente encontró asociación entre intento suicida y calidad de vida en hombres ${ }^{32}$; un elemento que puede haber influido es que la proporción de estudiantes que reportaron intento de suicidio fue menor en el presente estudio (3.0\% en mujeres y $1.1 \%$ en hombres) que en el anterior ( $9.7 \%$ en mujeres y $5.2 \%$ en hombres) y que la asociación ocurrió en población con promedio de edad menor donde podría asociarse de manera diferente.

También podría considerarse como posible explicación de la no asociación entre intento de suicidio y calidad de vida al hecho de que el cuestionario indagó sobre los CRS llevados a cabo en los últimos 12 meses mientras que se preguntó sobre la CVRS experimentada en los últimos 7 días. Esta diferencia en el periodo de tiempo considerado, podría implicar que la relación entre 
Tabla 5. Modelos de Regresión Logística de comportamientos de riesgo de suicidio y dominios de CVRS ajustados por variables de control de estudiantes mexicanos que ingresaron a un campus universitario en el año $2016(\mathrm{n}=1229)$.

\begin{tabular}{|c|c|c|c|c|c|c|c|c|c|}
\hline \multirow{2}{*}{ Variables de exposición y control } & \multicolumn{3}{|c|}{ Entorno escolar } & \multicolumn{3}{|c|}{ Aceptación social } & \multicolumn{3}{|c|}{ Recursos económicos } \\
\hline & $\%$ & OR & IC $95 \%$ & $\%$ & OR & IC 95\% & $\%$ & OR & IC 95\% \\
\hline Desesperanza $\left(\text { sí }^{\prime} 1 ; \text { no }=0\right)^{\mathrm{b}}$ & 34.1 & 3.0 & $1.4-6.4^{\star *}$ & 24.2 & 1.9 & $1.3-2.7^{\star \star}$ & 16.0 & 1.3 & $.9-1.8$ \\
\hline Ideación suicida $(s i ́=1 ; \text { no }=0)^{b}$ & 15.9 & 1.5 & $0.4-6.6$ & 10.5 & 1.7 & $.8-3.7$ & 6.4 & 1.6 & $.7-3.8$ \\
\hline Planeación suicida $(s i ́=1 ; \text { no }=0)^{b}$ & 9.1 & 0.5 & $0.1-2.1$ & 8.7 & 1.4 & $.6-3.2$ & 4.9 & 1.0 & $.5-2.4$ \\
\hline Intento suicida $\left(s^{\prime}=1 ; \text { no }=0\right)^{\mathrm{b}}$ & 11.4 & 3.8 & $0.8-18.2$ & 5.9 & 1.9 & $.7-5.0$ & 3.2 & 1.6 & $.5-4.5$ \\
\hline Abuso sexual $\left(\text { sí }^{\prime}=1 ; \text { no }=0\right)^{c}$ & 6.8 & 5.1 & $1.0-25.2^{*}$ & 2.4 & 1.8 & $.6-5.7$ & 1.5 & 1.6 & $.5-5.6$ \\
\hline Violencia física $\left(\mathrm{sí}^{-}=1 ; \text { no }=0\right)^{\mathrm{c}}$ & 9.1 & 1.2 & $0.4-3.8$ & 10.5 & 2.1 & $1.3-3.6^{\star *}$ & 6.7 & 1.3 & $.7-2.1$ \\
\hline $\begin{array}{l}\text { Consumo de tabaco }(\geq 6 \text { cigarrillos } \\
\text { diarios }=1 ; \leq 5=0)^{\text {a }}\end{array}$ & 2.3 & 1.0 & $0.1-13.3$ & 0.3 & 0.2 & $.0-1.9$ & 0.5 & 0.6 & $.1-2.8$ \\
\hline $\begin{array}{l}\text { Consumo de alcohol ( } \geq 5 \text { bebidas en } \\
2 \text { horas }=1 ; \leq 4=0)^{\text {a }}\end{array}$ & 36.4 & 0.8 & $0.4-1.7$ & 35.2 & 1.1 & $.8-1.5$ & 29.9 & 0.9 & $.7-1.1$ \\
\hline $\begin{array}{l}\text { Consumo de marihuana }(33 \text { veces }= \\
1 ; \leq 2=0)^{c}\end{array}$ & 13.6 & 2.7 & $1.0-7.8$ & 7.3 & 1.8 & $.9-3.2$ & 5.2 & 1.5 & $.8-2.8$ \\
\hline Trabajo $($ sí $=1 ;$ no $=0)$ & 45.5 & 1.5 & $0.8-2.8$ & 32.4 & 1.1 & $.8-1.4$ & 34.1 & 1.4 & $1.1-1.8^{*}$ \\
\hline Género $($ mujer $=1 ;$ hombre $=0)$ & 45.5 & 0.4 & $0.2-.8^{\star \star}$ & 64.5 & 1.1 & $.8-1.5$ & 63.3 & 1.0 & $.8-1.3$ \\
\hline Edad $(15-17=1 ; 18$ y $19=0)$ & 86.4 & 0.4 & $0.1-.9^{*}$ & 92.3 & 1.0 & $.6-1.6$ & 92.7 & 1.0 & $.7-1.6$ \\
\hline Discapacidad $($ sí $=1 ;$ no $=0$ ) & - & - & - & 6.3 & 1.0 & $.6-1.8$ & 5.8 & 1.0 & $.6-1.7$ \\
\hline $\begin{array}{l}\text { Nivel socioeconómico }(\text { medio bajo y } \\
\text { trabajador }=1 ; \text { medio alto y alto }=0 \text { ) }\end{array}$ & 47.7 & 1.2 & $0.6-2.3$ & 46.3 & 1.2 & $.9-1.5$ & 54.9 & 2.6 & $\begin{array}{c}2.1- \\
3.3^{* * *}\end{array}$ \\
\hline
\end{tabular}

Nota: \% = proporción de personas que tienen categoría de riesgo en las variables de exposición o control y una puntuación inferior en los dominios de calidad de vida relacionada con la salud (CVRS). Para los dominios de CVRS se consideró inferior una puntuación igual o menor a 42. Las tablas 3,4 y 5 corresponden a distintos dominios de CVRS. El guion (-) significa que no se registraron casos. ${ }^{a}$ Durante los últimos 30 días; ${ }^{\mathrm{b}}$ Durante los últimos 12 meses; ${ }^{\mathrm{c}}$ Durante la vida. ${ }^{\star} p<.05 ;{ }^{* *} p<.01 ;{ }^{\star * *} p<.001$

el CRS y la CVRS podría cobrar distintos significados dependiendo del grado de concordancia cronológica que tuvieran ambas medidas, sin embargo esta hipótesis no se sostiene si se consideran los hallazgos de la investigación referida a nivel preparatoria ${ }^{32}$ en la cual sí se asoció el intento suicida y la calidad de vida aun cuando tampoco había concordancia cronológica.

Algunas limitaciones de este estudio podrían deberse a su diseño, ya que los estudios de corte transversal no pueden explicar la dirección de las variables y por tanto no es posible explicar la causalidad, sin embargo, los estudios transversales aportan para explicar en qué medida las variables se asocian una a otras y lo hacen en un entorno real donde las personas están influenciadas por múltiples variables. Por tratarse de un instrumento de autoreporte que implica rescatar recuerdos de distintos periodos se puede presentar sesgo de memoria, sin embargo por tratarse de una población joven ${ }^{53}$ y por indagar temas de relevancia para la persona el sesgo podría ser menor. Los ítems indagan en periodos de tiempo que no obedecen a un patrón uniforme, pero esto se debe a que los comportamientos con una frecuencia menor requieren tiempos de observación más amplios que aquellos con una mayor frecuencia. Algunos estudiantes no contestaron completo el cuestionario y estos pudieron haber tenido características importantes, como haber presentado alguno de los comportamientos de riesgo o una calidad de vida baja. No obstante, pudimos recoger una muestra amplia de estudiantes por lo que consideramos que los datos mostrados representan a una gran parte de la población estudiada. El instrumento que utilizamos es un instrumento diseñado para la vigilancia en grandes poblaciones por lo cual no tiene por objetivo profundizar en la complejidad de la elaboración de los planes suicidas, sin embargo, fue un instrumento adecuado para el caso del proyecto al que pertenece este estudio que buscaba evaluar a todos los estudiantes que ingresan a un centro universitario.

En conclusión, en estudiantes universitarios mexicanos la CVRS se asocia a los CRS aún después de ajustar por consumo de sustancias, violencia y variables sociodemográficas. La des- 
esperanza se asoció negativamente a los distintos dominios de la CVRS de los estudiantes. A partir de nuestros hallazgos consideramos que los programas de prevención del suicidio no solo deberían estar dirigidos hacia los comportamientos más proximales como la ideación o intento suicida, sino a la desesperanza, así se podría no solo abonar a la prevención del suicidio, sino también a favorecer que el adolescente tenga una calidad de vida mejor.

\section{Colaboradores}

CA Hidalgo-Rasmussen: trabajó en la concepción, diseño, aplicación de instrumentos, análisis y redacción final del estudio y aprobación de la versión final; YV Chávez-Flores en el análisis, discusión, revisión crítica del artículo, redacción final y aprobación de la versión final; LY Yanez-Peñúñuri en interpretación de los datos, borrado del artículo, aprobación de la versión final; SRM Navarro en el análisis e interpretación de datos, revisión crítica del artículo y aprobación de la versión final. 


\section{Referências}

1. Organización Mundial de la Salud (OMS). Prevención del suicido: un instrumento para trabajadores de atención primaria de salud [Internet]. Ginebra: OMS; 2000. [acceso 2014 Oct 22]. Disponible en: http:// www.who.int/mental_health/media/primaryhealthcare_workers_spanish.pdf

2. Instituto Nacional de Estadística y Geografía. Estadísticas a propósito del Día Mundial para la prevención del Suicidio (10 de Septiembre) [Internet]. 2016. [acceso 2014 Oct 22]. Disponible en: http:// www.inegi.org.mx/saladeprensa/aproposito/2016/ suicidio2016_0.pdf

3. Organización Mundial de la Salud (OMS). Prevención del suicidio: un imperativo global [Internet]. Washington: OMS; 2014 [acceso 2014 Oct 22]. Disponible en: http://apps.who.int/iris/bitstre am/10665/136083/1/9789275318508_spa.pdf?ua=1

4. Bridge JA, Goldstein TR, Brent DA. Adolescent suicide and suicidal behavior. J Child Psychol Psychiatry 2006; 47(3-4):372-394.

5. Beck AT, Kovacs M, Weissman A. Assessment of suicidal intention: the Scale for Suicide Ideation. J Consult Clin Psychol 1979; 47(2):343-352.

6. Nock MK, Borges G, Bromet EJ, Cha CB, Kessler RC, Lee S. Suicide and suicidal behavior. Epidemiol Rev 2008; 30(1):133-154.

7. Hidalgo-Rasmussen CA. De los comportamientos de riesgo a la calidad de vida de los adolescentes. Cd. Guzmán: Universidad de Guadalajara; 2008.

8. Calvo J, Sánchez R, Tejada P. Prevalencia y Factores Asociados a Ideación Suicida en Estudiantes Universitarios. Rev Salud Publica 2003; 5(2):123-143.

9. Sánchez-Teruel D, García-León A, Muela-Martínez JA. Relación entre alta ideación suicida y variables psicosociales en estudiantes universitarios. Electron $J$ Res Educ Psychol 2013; 11(2):429-450.

10. Davaasambuu S, Batbaatar S, Witte S, Hamid P, Oquendo MA, Kleinman M, Olivares M, Gould M. Suicidal Plans and Attempts Among Adolescents in Mongolia. Crisis 2017; 38(5):330-343.

11. Espinoza-Gómez F, Zepeda-Pamplona V, Bautista-Hernández V, Hernández-Suárez CM, Newton-Sánchez OA, Plasencia-García GR. Violencia doméstica y riesgo de conducta suicida en universitarios adolescentes. Salud Publica Mex 2010; 52(3):213219.

12. Centers for Disease Control and Prevention (CDC). Measuring Healthy Days [Internet]. Atlanta: CDC; 2000 [acceso 2017 Mar 13]. 44 p. Disponible en: https://www.cdc.gov/hrqol/pdfs/mhd.pdf

13. Villalonga-Olives E, Kawachi I, Almansa J, von Steinbüchel $\mathrm{N}$. Longitudinal changes in health related quality of life in children with migrant backgrounds. PLoS One 2017; 12(2):e0170891.

14. Villalonga-Olives E, Rojas-Farreras S, Vilagut G, Palacio-Vieira JA, Valderas J, Herdman M, Ferrer M, Rajmil L, Alonso J. Impact of recent life events on the health related quality of life of adolescents and youths: the role of gender and life events typologies in a follow-up study. Health Qual Life Outcomes 2010; 8:71.

15. Banks BA, Barrowman NJ, Klaassen R. Health-related Quality of Life: Changes in Children Undergoing Chemotherapy. J Pediatr Hematol Oncol 2008; 30(4):292-297.
16. McDougall J, Wright V, Schmidt J, Miller L, Lowry $\mathrm{K}$. Applying the ICF framework to study changes in quality-of-life for youth with chronic conditions. Dev Neurorehabil 2010; 14(1):41-53.

17. Goldenberg M, Danovitch I, IsHak WW. Quality of life and smoking. Am J Addict 2014; 23(6):540-562.

18. Stewart SH, Hutson A, Connors GJ. Exploration of the Relationship between Drinking Intensity and Quality of Life. Am J Addict 2006; 15(5):356-361.

19. Hidalgo-Rasmussen C, Hidalgo-San Martín A, Rasmussen-Cruz B, Hernández-Mejía R, Santollyo-Télles F. Comportamiento de riesgo por consumo de alcohol y calidad de vida en estudiantes universitarios. Rev Mex del Inst Mex del Seguro Soc [Internet]. 2009 [acceso 2014 Jul 9]; 47(Supl. 1):S7-S12. Disponible en: http://revistamedica.imss.gob.mx/index php?option $=$ com_multicategories\&view $=$ article\&id $=879$ : comportamiento-de-riesgo-por-consumode-alcohol-y-calidad-de-vida-en-estudiantes-universitarios\&Itemid $=599$

20. Goldenberg M, IsHak WW, Danovitch I. Quality of life and recreational cannabis use. Am J Addict 2016; 26(1):8-25.

21. Lev-wiesel R. Quality of Life in Adult Survivors of Childhood Sexual Abuse Who Have Undergone Therapy. J Child Sex Abus 2000; 9(1):1-13.

22. Sørensen J, Kruse M, Gudex C, Helweg-Larsen K, Bronnum-Hansen H. Physical violence and health-related quality of life: Danish cross-sectional analyses. Health Qual Life Outcomes 2012; 10:113.

23. Gandelman N, Piani G. Quality of Life Satisfaction Among Workers and Non-Workers in Uruguay. Soc Indic Res 2012; 111(1):97-115.

24. Michel G, Bisegger C, Fuhr DC, Abel T, The KIDSCREEN group. Age and gender differences in health-related quality of life of children and adolescents in Europe: a multilevel analysis. Qual Life Res 2009; 18(9):1147-1157.

25. Simões C, Santos S. Comparing the quality of life of adults with and without intellectual disability. J Intellect Disabil Res 2016; 60(4):378-388.

26. Mielck A, Vogelmann M, Leidl R. Health-related quality of life and socioeconomic status: inequalities among adults with a chronic disease. Health Qual Life Outcomes 2014; 12:58.

27. Lester D. Hopelessness in undergraduate students around the world: A review. J Affect Disord 2013; 150(3):1204-1208.

28. Lester D. Hopelessness in adolescents. J Affect Disord 2015; 173:221-225.

29. Osnaya MC, Pérez JCR. La desesperanza de riesgo en jóvenes mexicanos y aspectos sociodemográficos asociados: diferencias por sexo. Psicol y Salud 2010; 20(2):195-201.

30. Vázquez-Vega D, Piña-Pozas M, González-Forteza C, Jiménez-Tapia A, Mondragón-Barrios L. La investigación sobre suicidio en México en el periodo 1980-2014: análisis y perspectivas. Acta Univ 2015; 25(2):62-69.

31. Pérez JCR, Osnaya MC. Ideación suicida y su relación con variables de identificación personal en estudiantes universitarios mexicanos. Rev Intercont psicol educ 2011; 13(2):9-30. 
32. Hidalgo-Rasmussen C, Hidalgo-San MA. Comportamientos de riesgo de suicidio y calidad de vida, por género, en adolescentes mexicanos, estudiantes de preparatoria. Cien Saude Colet 2015; 20(11):34373445.

33. Centers for Disease Control and Prevention (CDC). 2015 National Youth Risk Behavior Survey. Atlanta: CDC; 2015.

34. Brener N, Kann L, McManus T, Kinchen S, Sundberg E, Ross J. Reliability of the 1999 Youth Risk Behavior Survey Questionnaire. J Adolesc Heal 2002; 31(4):336342.

35. Brener N, Billy J, Grady W. Assessment of factors affecting the validity of self-reported health-risk behavior among adolescents: evidence from the scientific literature. J Adolesc Heal 2003; 33(6):436-457.

36. Hidalgo-Rasmussen CA, Rajmil L, Espinoza RM. Adaptación transcultural del cuestionario KIDSCREEN para medir calidad de vida relacionada con la salud en población mexicana de 8 a 18 años. Cien Saude Colet 2014; 19(7):2215-2224.

37. Brener ND, Kann L, McManus T, Kinchen SA, Sundberg EC, Ross JG. Reliability of the 1999 youth risk behavior survey questionnaire. J Adolesc Health 2002; 31(4):336-342.

38. Rivas-Torres R, Bianchi-Águila R. El nivel socioeconómico en la investigación. Rev la Asoc Mex Enfermedades metabólicas y Obes 1991; 2(1):44-45.

39. Revicki DA, Cella D, Hays RD, Sloan JA, Lenderking WR, Aaronson NK. Responsiveness and minimal important differences for patient reported outcomes. Health Qual Life Outcomes 2006; 4:70.

40. World Medical Association. World Medical Association Declaration of Helsinki: ethical principles for medical research involving human subjects. Jama 2013; 310(20):2191-2194.

41. Beck AT, Steer RA, Kovacs M, Garrison B. Hopelessness and eventual suicide: a 10-year prospective study of patients hospitalized with suicidal ideation. Am J Psychiatry 1985; 142(5):559-563.

42. Taliaferro LA, Rienzo BA, Pigg RM, Miller MD, Dodd VJ. Associations Between Physical Activity and Reduced Rates of Hopelessness, Depression, and Suicidal Behavior Among College Students. J Am Coll Health 2009; 57(4):427-436.

43. Rosellini AJ, Bagge CL. Temperament, Hopelessness, and Attempted Suicide: Direct and Indirect Effects. Suicide Life-Threatening Behav 2014; 44(4):353-361.

44. Baaba G, Anakwah N, Owusu J. "Unattractive, So Hopeless?" Feelings of Physical Unattractiveness and Hopelessness among Senior High Students. Eur Res 2016; 110(9):500-507.
45. Becker-Weidman EG, Reinecke MA, Jacobs RH, Martinovich Z, Silva SG, March JS. Predictors of Hopelessness Among Clinically Depressed Youth. Behav Cogn Psychother 2009; 37(3):267-291.

46. Shek DTL, Li X. Perceived School Performance, Life Satisfaction, and Hopelessness: A 4-Year Longitudinal Study of Adolescents in Hong Kong. Soc Indic Res 2015; 126(2):921-934.

47. Kashani JH, Suarez L, Allan WD, Reid JC. Hopelessness in Inpatient Youths: A Closer Look at Behavior, Emotional Expression, and Social Support. J Am Acad Child Adolesc Psychiatry 1997; 36(11):1625-1631.

48. Kwok SYCL, Shek DTL. Hopelessness, Parent-Adolescent Communication, and Suicidal Ideation among Chinese Adolescents in Hong Kong. Suicide Life-Threatening Behav 2010; 40(3):224-233.

49. LaRusso MD, Romer D, Selman RL. Teachers as Builders of Respectful School Climates: Implications for Adolescent Drug Use Norms and Depressive Symptoms in High School. J Youth Adolesc 2007; 37(4):386398.

50. Kandel DB, Raveis VH, Davies M. Suicidal ideation in adolescence: Depression, substance use, and other risk factors. J Youth Adolesc 1991; 20(2):289-309.

51. Bearman PS, Moody J. Suicide and friendships among American adolescents. Am J Public Health 2004; 94(1):89-95.

52. Silverman MM, Berman AL, Sanddal ND, O'Carroll PW, Joiner TE. Rebuilding the Tower of Babel: A Revised Nomenclature for the Study of Suicide and Suicidal Behaviors Part 1: Background, Rationale, and Methodology. Suicide Life-Threatening Behav 2007; 37(3):248-263.

53. Simón T, Gallego-Largo TR, Suengas AG. Memoria y envejecimiento: Recuerdo, reconocimiento y sesgo positivo. Psicothema 2009; 21(3):409-415.

Artigo apresentado em 05/07/2017

Aprovado em 12/03/2018

Versão final apresentada em 15/03/2018 